\title{
Evaluating the role of intestinal parasites in the high rates of irritable bowel syndrome in South America: a pilot study
}

\author{
George Vasquez-Rios $^{1,2}$, Jorge D. Machicado ${ }^{2,3}$, Maria T. Gamero ${ }^{1}$, Adriana Pezua $^{1}$, Angel B. Betancourt ${ }^{1}$, \\ Angelica Terashima ${ }^{1,2}$ and Luis A. Marcos ${ }^{2,4}$ \\ ${ }^{1}$ Facultad de Medicina, Alberto Hurtado, Universidad Peruana Cayetano Heredia, Lima, Peru; \\ ${ }^{2}$ Instituto de Medicina Tropical Alexander von Humboldt, Hospital Nacional Cayetano Heredia, Lima, Peru; \\ ${ }^{3}$ Division of Gastroenterology, Hepatology and Nutrition, University of Pittsburgh Medical Center, Pittsburgh, PA, USA; \\ ${ }^{4}$ Department of Medicine and Molecular Genetics and Microbiology, Stony Brook University, Stony Brook, New York, USA
}

\begin{abstract}
There is limited data on the role of intestinal parasites in irritable bowel syndrome (IBS) in South America. We evaluated the association between intestinal parasitism and IBS status in Peru. Intestinal parasites were detected in $43 \%$ of the IBS cases and in $51 \%$ of the controls $(\mathrm{P}=0.4)$. After excluding those infected by any parasite, the IBS prevalence remained high $(22 \% ; \mathrm{P}=0.7)$. No statistically significant difference was noted between IBS cases and controls in terms of monoparasitism, biparasitism or multiparasitism. Furthermore, the protist Blastocystis sp. was inversely associated with IBS.
\end{abstract}

Keywords: soil-transmitted helminths, Blastocystis sp., functional gastrointestinal diseases

Irritable bowel syndrome (IBS) is a functional gastrointestinal (GI) disorder, characterised by abdominal discomfort, bloating and altered bowel habits. Recently, the world-wide pooled prevalence of IBS was estimated to be $11 \%$ and in a separate analysis, South America was found to be the region with the highest occurrence at 21\% (Lovell and Ford 2012).

Precipitation, temperature and poor sanitary conditions encountered in tropical regions such as South America may contribute to the high prevalence of intestinal parasites such as Ascaris lumbricoides Linnaeus, 1758, Strongyloides stercoralis (Bavay, 1876), hookworm, Trichuris trichiura (Linnaeus, 1771), Hymenolepis nana (Stiles, 1906), Entamoeba histolytica Schaudinn, 1903 and Giardia lamblia (Kunstler, 1882), which present a wide array of GI symptoms, that may mimic those reported by IBS patients from endemic settings (Stark et al. 2007).

The role of intestinal parasitic infections in IBS is unclear, especially in South America where the prevalence of IBS and intestinal parasitism are both high. We hypothesised that the high rates of IBS described are associated with an unmeasured infection by these organisms. Therefore, we estimated the prevalence of IBS in individuals with and without parasites in Peru. In a secondary analysis, we evaluated the association between IBS status and being infected by a single parasite, biparasitism or multiparasitism.

\section{MATERIALS AND METHODS}

\section{Setting and population}

The present study was conducted from December 2012 to June 2013 among two army bases in Peru: Fuerte Soldado Alfredo Vargas Guerra (FSAVG) located in Iquitos (Amazon basin) and Cuartel General del Ejercito (CGE), located in Lima (coast). The study population was composed by 435 active duty soldiers; 250 from FSAVG and 185 corresponding to CGE. Military members were invited to participate in this study and were requested to provide one fresh stool sample. They also completed an anonymous survey that included the validated Spanish version of the Rome III questionnaire, in addition to socio-demographic and clinical variables.

\section{Study design}

We used a cross-sectional design to describe the rates of IBS in infected and non-infected subjects. Then, among participants we applied a case-control design to elucidate the potential risk for IBS with any single parasitic infection, biparasitism or multiparasitism. Participants were selected by convenience, on the basis of their presence in the military base and their willingness to participate in the study. Participants with a known GI disease such as inflammatory bowel disease (IBD), celiac disease (CD) or colon cancer were excluded from the study. A case was defined as a subject who was diagnosed with IBS through the Rome III criteria. Moreover, a control was a subject who did not fulfill the diagnosis

Address for correspondence: G. Vasquez-Rios, Laboratory of Parasitology, Instituto de Medicina Tropical Alexander von Humboldt, Universidad Peruana Cayetano Heredia, Av. Honorio Delgado 264, Lima 15102, Peru. Phone: +51012736738; Fax: +51012754735; E- mail: george.vasquez@upch.pe 
Table 1. Epidemiological characteristics of irritable bowel syndrome (IBS) cases and controls.

\begin{tabular}{lcccc}
\hline & IBS cases $(\mathrm{n}=37)$ & Controls $(\mathrm{n}=148)$ & P-value & Total $(\mathrm{n}=185)$ \\
\hline Age, median & $20( \pm 2.66)$ & $20( \pm 2.61)$ & & \\
Female gender, $\%$ & $30 \%(11)$ & $30 \%(44)$ & 1 & $30 \%(55)$ \\
Weight loss, $\%$ & $60 \%(22)$ & $31 \%(46)$ & 0.01 & $37 \%(68)$ \\
Haematochezia, $\%$ & $27 \%(10)$ & $7 \%(10)$ & $<0.001$ & $11 \%(20)$ \\
\hline
\end{tabular}

Table 2. Parasitological screening among irritable bowel syndrome (IBS) cases and controls.

\begin{tabular}{lcccc}
\hline Intestinal parasite & IBS case $(\mathrm{n}=37)$ & Control $(\mathrm{n}=148)$ & P-value & OR (95\% IC) \\
\hline Monoparasitism & $16 \%(6)$ & $28 \%(42)$ & 0.2 & \\
Biparasitism & $14 \%(5)$ & $14 \%(21)$ & 0.3 & \\
Multiple pathogenic parasites $(\geq 3)$ & $14 \%(5)$ & $8 \%(12)$ & 0.5 & \\
Ascaris lumbricoides Linnaeus, 1758 & $16 \%(6)$ & $16 \%(24)$ & 0.8 & \\
Hookworm (Ancylostomidae) & $30 \%(11)$ & $23 \%(34)$ & 0.4 & \\
Trichuris trichiura (Linnaeus, 1771) & $14 \%(5)$ & $11 \%(16)$ & 0.5 & \\
stercoralis (Bavay, 1876) & $19 \%(7)$ & $14 \%(20)$ & 0.3 & \\
Giardia lamblia (Kunstler, 1882) & $11 \%(4)$ & $18 \%(27)$ & 0.9 & \\
Hymenolepis nana (Stiles, 1906) & $3 \%(1)$ & $2 \%(3)$ & 0.5 & $0.28(0.12-0.64)$ \\
Blastocystis sp. $\dagger$ & $49 \%(18)$ & $71 \%(105)$ & $<0.01$ & \\
\hline
\end{tabular}

$\dagger$ Blastocystis sp. was considered a controversial protist in this study; OR - Mantel-Haenszel odds ratios.

of IBS. In addition, information about weight loss ( $>10 \%$ of body weight in the last 6 months) as well as haematochezia was collected in both groups. Cases and controls were matched by gender and age ( \pm 2 years) in a 1 (case): 4 (control) ratio.

\section{Laboratory analysis}

Stool samples were collected by using labelled plastic containers without preservatives and examined in less than 6 hours at the Institute of Tropical Medicine Alexander von Humboldt, Universidad Peruana Cayetano Heredia. Stools samples were analysed macroscopically for consistency and presence of blood, mucus or adult helminth parasites. Microscopic examination included the Pavlova culture (PC) for the diagnosis of Blastocystis sp. and two stool concentrations techniques to maximise the detection of both soil-transmitted helminths (STH) [Spontaneous Sedimentation in Tube Technique (SSTT)] and Strongyloides stercoralis [Lumbreras' cup modified Baermann's Technique (MBT)].

Detection of Ascaris lumbricoides, S. stercoralis, hookworms, Trichuris trichiura, Hymenolepis nana or Giardia lamblia by any of the parasitological techniques was considered as a pathogenic infection on the basis of internationally accepted classification (Centers for Disease Control and Prevention 2011). Moreover, we considered a positive stool sample for the controversial protist (Blastocystis sp.) once it was detected by either SSTT or PC technique. As the prevalence of Entamoeba spp. was very low in both cases and controls, we did not perform further analysis to differentiate it from Entamoeba dispar (Brumpt, 1925)/Entamoeba histolytica and it was not included in the study.

\section{Statistical analysis}

Data were analysed with STATA 12.1 (StataCorp, College Station, Texas, USA). Frequency rates were calculated for discrete variables. For continuous variables, mean or median with standard deviation (SD) were presented. We estimated a sample size of 37 cases and 148 controls to detect a statistically significant difference between both groups at a significance level of
$5 \%$ and a power of $90 \%$. Bivariate analysis was performed with Chi-square test for discrete variables, whereas t-test was used for continuous variables. Mantel-Haenszel odds ratios (OR) were calculated to determine the association between IBS and intestinal parasite infection. A $\mathrm{P}$ value $<0.05$ was considered as statistically significant.

\section{Ethics}

The Institutional Review Board of Ethical Committee from Universidad Peruana Cayetano Heredia at Lima, Peru approved the study (IRB No. 60584). Characteristics of this study (duration, procedures, benefits and minimal risks) as well as confidentiality were explained prior to enrollment. Consent was obtained from all participants and the results were reported to the local health authorities.

\section{RESULTS}

Out of 435 individuals, a total of 185 (43\%) participated in this study, including 95 adult soldiers from Lima and 90 from Iquitos. No individual presented a concomitant GI condition such as IBD, CD or colon cancer in either the case or control group. Participants were more frequently men $(70 \%)$ and the age ranged between $18-28$ years (mean: $20 \pm 2.66$ ). Clinical features such as weight loss in the last six months and haematochezia were observed in $60 \%(n=22)$ and $27 \%(n=10)$ of the IBS cases, respectively (Table 1).

The overall prevalence of IBS was $20 \%(n=37 ; 95 \%$ CI: 14-26\%). In further analysis, after excluding subjects infected with any parasite, the prevalence of IBS was $22 \%$ $(\mathrm{n}=21 ; \mathrm{P}=0.7)$. Intestinal parasites were detected in $43 \%$ $(n=16)$ participants with IBS and in $51 \%(n=75)$ of the control group $(\mathrm{P}=0.4)$. There was no statistically significant difference between the control group and IBS participants in terms of monoparasitism $(\mathrm{P}=0.2)$, biparasitism $(\mathrm{P}=0.3)$ or multiparasitism $(\mathrm{P}=0.5)$. In addition, the pro- 
tist Blastocystis sp. was present in $49 \%(\mathrm{n}=18)$ of the cases and $71 \%(\mathrm{n}=105)$ of the controls (OR: $0.28 ; 95 \% \mathrm{CI}$ : 0.12-0.64; $\mathrm{P}<0.01$ ) (Table 2).

\section{DISCUSSION}

The prevalence of IBS in this specific Peruvian population was high $(20 \%)$ and is in agreement with previous studies in the region, in which the IBS rates ranged between 15-30\% (Lovell and Ford 2012). Differences may be attributed to the heterogeneity of diagnostic criteria, limited sample size and distinct risk factors for IBS between study populations.

We found that after excluding individuals with concomitant parasitic infection, the prevalence of IBS did not change. Furthermore, the lack of difference may be due to an equal distribution of intestinal parasites among both cases and controls. Based on our results, parasitological evaluation could not alter the diagnosis of IBS in endemic populations either in clinical practice or in epidemiological studies. Nonetheless, additional evaluation in large population-based studies is needed to confirm this finding. It is unknown whether the infection by helminths or protists during childhood could modify the gut microbiome and predispose some individuals for developing IBS later during adulthood (Rigsbee et al. 2012). Further studies will need to address the effect of intestinal parasitism in microbiome modulation, with special attention to the disability-adjusted life years in infected children.

With regard to the potential role of parasites in IBS status, we found that the number of parasites was not associated with IBS. Interestingly, the same findings have been reported in large population-based studies in Nicaragua (Morgan et al. 2012) and more recently in Denmark (Krogsgaard et al. 2015). Also, in concordance with previous reports, we found that that Giardia lamblia carriage among IBS subjects is non-negligible, although it was not associated to this condition. This observation deserves special attention for some reasons. For example, G. lamblia can cause clinical symptoms that may be difficult to differentiate from those reported by IBS patients (Grazioli et al. 2006). In addition, this parasite has been implicated in the appearance of IBS after eradication (post-infectious IBS) (Hanevik et al. 2014). Hence, infected participants need follow-up owed to the increased risk of developing IBS in the future, as well as other complications such as chronic fatigue syndrome, interstitial cystitis, etc.

The role of Blastocystis in IBS is controversial. Giacometti et al. (1999) and Yakoob et al. (2004) described a positive association between Blastocystis and the IBS diagnosis in Italy and Pakistan, respectively. More recently, this organism along with Dientamoeba fragilis (Jeeps et Dobell, 1918) have been detected to be more prevalent in patients fulfilling the IBS diagnosis compared to controls, by using advanced techniques (Yakoob et al. 2010). In contrast, studies in Thailand, Turkey, Mexico and Denmark have shown an inverse correlation, similar to our study (Surangsrirat et al. 2010, Cekin et al. 2012, Krogsgaard et al. 2015). Three common limitations have been present in several studies. First, controls were participants with GI disorders other than IBS and the composition of the intestinal microbiome may be compromised, including the absence of Blastocystis (dysbiosis). Second, most studies have not reported the exclusion of patients with previous exposure to antibiotics such as trimethoprim/sulfamethoxazole and metronidazole, which can decrease carriage of Blastocystis and potentially cause a microbiome disturb. Third, only few studies have included a subtype analysis of Blastocystis, which may have different clinical implications in humans.

There are several limitations in our study, such as a small sample size and the lack of randomisation. Also, our study population was under-represented by women, which is a known risk factor for IBS. Furthermore, including participants from two different locations in Peru may limit our external validity. Moreover, given the high prevalence of intestinal parasites in this area, a true difference between cases and controls in terms of parasitic carriage could have been missed owed to the sample size. Despite the use of several parasitological techniques to maximise the detection of parasites, by using only one fresh stool sample, false negative results are expected owed to the irregular shedding of eggs and cysts. Although culture is a high sensible diagnostic method for Blastocystis, recently time recovery of this organism has been demonstrated to be different between IBS patients and IBS negative individuals, possibly due to an altered intestinal environment. This may influence the generation time (strain selection). Finally, we did not perform molecular analysis for Blastocystis subtyping and we cannot extend our results towards to a protective role of this organism.

In summary, in this specific population from the tropics, the prevalence of IBS was high and intestinal parasitic infection was not associated with this infection level. In terms of parasite carriage, no organism was associated with IBS. In contrast, Blastocystis was negatively associated with IBS and this finding warrants additional analysis. To the best of our knowledge, this is the first study in South America that assesses a potential role for intestinal parasites in IBS. Our results are exploratory and further studies are needed in this region to assess the role and epidemiological association between intestinal parasites and IBS.

Acknowledgements. We are grateful for the collaboration of military personnel from the military base Fuerte Soldado Alfredo Vargas Guerra (FSAVG) and Cuartel General del Ejercito (CGE) and their willingness to participate in the present study. 


\section{REFERENCES}

Cekin A.H., Cekin Y., Adakan Y., Tasdemir E., Koclar F.G., YolCUlar B.O. 2012: Blastocystosis in patients with gastrointestinal symptoms: a case-control study. BMC Gastroenterol. 12: 122 .

Centers for Disease Control and Prevention 2011: DPDxLaboratory Identification of Parasitic Diseases of Public Health Concern. Atlanta, GA: U.S. Department of Global Health - Division of Parasitic Diseases and Malaria.

Giacometti A., Cirioni O., Fiorentini A., Fortuna M., SCAlise G. 1999: Irritable bowel syndrome in patients with Blastocystis hominis infection. Eur. J. Clin. Microbiol. Infect. Dis. 18: 436-439.

Grazioli, B, Matera G., Laratta C., Schipani G., GuarnieRi G., Spiniello E., Imeneo M., Amorosi A., Focì A., LuzZA F. 2006: Giardia lamblia infection in patients with irritable bowel syndrome and dyspepsia: a prospective study. World. J. Gastroenterol. 12: 1941-1944.

Hanevik K., Wensaas K.A., Rortveit G., Eide G.E., Mørch K., LANGELAND N. 2014: Irritable bowel syndrome and chronic fatigue 6 years after Giardia infection: a controlled prospective cohort study. Clin. Infect. Dis. 59: 1394-1400.

Krogsgaard L.R., Engsbro A.L., Stensvold C.R., Nielsen H.V., Bytzer P. 2015: The prevalence of intestinal parasites is not greater among individuals with irritable bowel syndrome: a population-based case-control study. Clin. Gastroenterol. Hepatol. 13: 507-513.

Received 11 September 2015

Accepted 12 October 2015
LOVELl R.M., Ford A.C. 2012: Global prevalence of and risk factors for irritable bowel syndrome: a meta-analysis. Clin. Gastroenterol. Hepatol. 10: 712-721

Morgan D.R., Benshoff M., Cáceres M., Becker-Dreps S., Cortes L., Martin C.F., Schmulson M., Peña R. 2012: Irritable bowel syndrome and gastrointestinal parasite infection in a developing nation environment. Gastroenterol. Res. Pract. 2012: 1-6.

Rigsbee L., Agans R., Shankar V., Kenche H., Khamis H.J., Michail S., Paliy O. 2012: Quantitative profiling of gut microbiota of children with diarrhea-predominant irritable bowel syndrome. Am. J. Gastroenterol. 107: 1740-1751.

Stark D., van Hal S., Marriott D., Ellis J., Harkness J. 2007: Irritable bowel syndrome: a review on the role of intestinal protozoa and the importance of their detection and diagnosis. Int. J. Parasitol. 37: 11-20.

Surangsrirat S., Thamrongwittawatpong L., Piyaniran W., Naaglor T., Khoprasert C., TaAmasri P., MungthIN M., Leelayoova S. 2010: Assessment of the association between Blastocystis infection and irritable bowel syndrome. J. Med. Assoc. Thai. 93 (Suppl 6): S119-S124.

Yakoob J., Jafri W., Beg M.A., Abbas Z., Naz S., Islam M., KHAN R. 2010. Blastocystis hominis and Dientamoeba fragilis in patients fulfilling irritable bowel syndrome criteria. Parasitol. Res. 107: 679-684.

YAKOоB J., JAFRI W., JAFRI N., KhaN R. 2004: Irritable bowel syndrome: in search of an etiology: role of Blastocystis hominis. Am. J. Trop. Med. Hyg. 70: 383-385.

Cite this article as: Vasquez-Rios G., Machicado J.D., Gamero M.T., Pezua A., Betancourt A.B., Terashima A., Marcos L.A. 2015: Evaluating the role of intestinal parasites in the high rates of irritable bowel syndrome in South America: a pilot study. Folia Parasitol. 62: 065. 